4

$14 *$ Corresponding author: Amar BOUAM, DVM, PhD. mailto:amarbouam@yahoo.fr

18 Phone number: +337582977 12

1. IHU Méditerranée Infection, Marseille, France. Marseille, France.

4. Centre Muraz, Bobo-Dioulasso, Burkina Faso.

\section{kingdom communication}

2. Aix Marseille Univ., IRD, MEPHI, IHU Méditerranée Infection, Marseille, France.

3. Aix Marseille Univ., IRD, AP-HM, SSA, VITROME, IHU Méditerranée Infection, 


\section{ABSTRACT}

\section{Background}

23 Mycobacterium ulcerans secrete a series of non-ribosomal-encoded toxins known as mycolactones that

24 are responsible for causing a disabling ulceration of the skin and subcutaneous tissues named Buruli

25 ulcer. The disease is the sole non-contagion among the three most common mycobacterial diseases in

26 humans. Direct contact with contaminated wetlands is a risk factor for Buruli ulcer, responsible for $M$.

27 ulcerans skin carriage before transcutaneous inoculation with this opportunistic pathogen.

\section{Methodology and principal findings}

29 In this study, we analysed the bacterial and fungal skin microbiota in individuals exposed to M. ulcerans

30 in Burkina Faso. We showed that M. ulcerans-specific DNA sequences were detected on the unbreached

31 skin of $6 / 52(11.5 \%)$ asymptomatic farmers living in Sindou versus $0 / 52(0 \%)$ of those living in the non-

32 endemic region of Tenkodogo. Then, we cultured the skin microbiota of asymptomatic M. ulcerans

33 carriers and negative control individuals, all living in the region of Sindou. A total of 84 different

34 bacterial and fungal species were isolated, 21 from $M$. ulcerans-negative skin samples, 31 from $M$.

35 ulcerans-positive samples and 32 from both. More specifically, Actinobacteria, Aspergillus niger and

36 Aspergillus flavus were significantly associated with $M$. ulcerans skin carriage. We further observed that

37 in vitro, mycolactones induced spore germination of A. flavus, attracting the fungal network.

\section{Conclusion}

39 These unprecedented observations suggest that interactions with fungi may modulate the outcome of $M$.

40 ulcerans skin carriage, opening new venues to the understanding of Buruli ulcer pathology, prophylaxis

41 and treatment of this still neglected tropical infection.

43 Keywords: Aspergillus spp., Aspergillus niger, Aspergillus flavus, Buruli ulcer, Mycobacterium

$44 \quad$ ulcerans, skin microbiota, fungi, Penicillium spp., Penicillium rubens. 


\section{Author summary}

46 Buruli ulcer is a chronic infectious disease caused by the environmental opportunistic pathogen

47 Mycobacterium ulcerans which secretes an exotoxin responsible for its pathogenicity. The reservoir and

48 sources of $M$. ulcerans in the environment remain elusive and its mode of transmission is unclear. To

49 acquire $M$. ulcerans infection, at least two conditions must be met, viable bacteria and a skin lesion as

50 demonstrated by experimental animal models. In this study, we showed that $M$. ulcerans specific DNA

51 sequences could be detected on the healthy skin of asymptomatic farmers living in one region of Burkina

52 Faso where Buruli ulcer cases had already been reported, but not in Buruli ulcer-free regions, suggesting

53 skin carriage after contacts with environmental sources. We also investigated the skin microbiota of $M$.

54 ulcerans carriers and found significant associations of some bacteria and fungi with skin carriage of $M$.

55 ulcerans. These associations may due to the effect of mycolactones on some fungi species. As we

56 showed previously with Mucor circinelloides and here with Aspergillus flavus. 


\section{Introduction}

58 Buruli ulcer is a disabling chronic disease of the skin and the subcutaneous tissues [1].

59 The disease has been reported since 1948 in several tropical regions [2], with the highest incidence being

60 observed in West African countries, including Côte d'Ivoire, Ghana, and Benin [3]. The causative agent

61 Mycobacterium ulcerans is a non-tuberculous mycobacterium harbouring a 174-kb circular virulence

62 plasmid pMUM [4-5] encoding three genes, $m l s \mathrm{~A} 1, m l s \mathrm{~A} 2$ and $m l s \mathrm{~B}$, responsible for the non-ribosomal

63 synthesis of mycolactone toxins, the main virulence factors of the pathogen [6]. This macrolide exotoxin

64 is secreted by a group of closely related non-tuberculous mycobacteria named Mycolactone Producing

65 Mycobacteria (MPM) [7-8]. Comparative analysis of whole genome sequences showed that MPM form a

66 single clonal group that evolved from a Mycobacterium marinum parent [9]. This group is divided into

67 three lineages, including frog and fish pathogens in one lineage [10-11], the Japanese strain M. ulcerans

68 subsp. shinshuense in a second lineage, while the third lineage includes a highly clonal group responsible

69 for Buruli ulcer in Africa and Australia [9]. Every strain synthesizes at least one of the eight congeners

70 of mycolactone A/B named mycolactone C, D, E, F, dia-F, S1 and S2 [12]. Each type of mycolactone

71 exhibits a variable degree of cytotoxicity, with mycolactone A/B being the most biologically active [13].

72 Indeed, this macrolide toxin is responsible for cytotoxic effects, namely, apoptosis and necrosis, in

73 addition to immunosuppressive and analgesic effects [12] after $M$. ulcerans has penetrated breached skin

74 to initiate discrete oedematous lesions that can evolve into typical Buruli ulcer lesions [14]. Currently,

75 the mode of transmission of M. ulcerans is debated. While it is clear that . ulcerans must be inoculated

76 through the skin to elicit Buruli ulcer, the specific role of aquatic bugs and mosquitoes as potential

77 vectors is debated along with the possibility of passive entry by any skin breach regardless of its cause. It

78 is not known whether M. ulcerans can colonize skin in asymptomatic populations exposed to

79 environments contaminated with $M$. ulcerans. Mycobacteria of the $M$. ulcerans group are known as

80 environmental organisms residing in poorly defined aquatic ecological niches where they could be part 
81 of an alimentary chain [15]. We previously reported that $M$. ulcerans could thrive in environmental

82 niches containing bacteria, fungi, algae and mollusks with which $M$. ulcerans may exchange nutrients

83 [16]. One may hypothesize that the cutaneous microbiota, which is part of the individual, partially

84 controls the expression of the M. ulcerans infection [17]. Indeed, bacteria and fungi present on skin

85 contaminated with $M$. ulcerans could interact with the pathogen, as previously shown with the

86 antagonism between Staphylococcus lugdunensis and Staphylococcus aureus in the nasal mucosa [18-

87 19].

88 To contribute to this medical debate, we have undertaken the first study to evaluate the possibility of

89 asymptomatic carriage of $M$. ulcerans on healthy skin, to characterize the cutaneous bacterial and fungal

90 microbiota associated with the asymptomatic carriage of $M$. ulcerans and to assess the biological

91 interactions between $M$. ulcerans and the skin microbiota.

\section{Methods}

\section{Ethics Statement}

95 This study was approved by the Centre MURAZ Ethics Committee, Burkina Faso, and reference

96 2018- 11/MS/SG/CM/CEI.

97 All adult subjects provided informed oral consent, and a parent or guardian of any child

98 participant provided informed oral consent on the child's behalf after explaining the merits of the

99 study. Written consent could not be obtained because the study was conducted among an illiterate

100 population. The research presents no more than minimal risk of harm to subjects and involves no

101 procedures for which written consent is normally required outside of the research context (Sec.

10256.109 IRB review of research).

103 Sample collection. Sampling was performed in two regions of Burkina Faso. Sindou is located in 104 the rural district of Niofila, Douna Department, Province of Léraba in the Cascades region in the 
105

106

107

108

109

110

111

112

113

114

115

southwest of Burkina Faso near the borders with Côte d'Ivoire and Mali. People sampled in this region are farmers in frequent contact with stagnant water because of their daily activities in rice, banana and vegetable cultivation areas that are irrigated from a nearby dam. Cases of Buruli ulcer have already been reported in this region [20]. Tenkodogo, a town located in the province of Boulgou and the Central-Eastern region of Burkina Faso, was used as a negative control region free of Buruli ulcer. Additionally, in this region, farmers working near two dams in rice and vegetable cultures were swabbed (the lower legs of the farmers were cleaned with water and then swabbed with sterile swabs (Deltalab, Barcelona, Spain) containing $1 \mathrm{~mL}$ of sterile $0.9 \%$ sodium chloride solution, the swabbed parts did not contain any visible lesions.). A total of 104 farmers were sampled, 52 farmers in each region. In Sindou region, the average age was 37 years, 30 females and 22 males. In Tenkodogo region the male/female ratio was 27/25 respectively with an average age of 20 years. One sample was taken per individual.

\section{Detection of $M$. ulcerans DNA.}

Real-time PCR amplifications. Total DNA from the samples and from a six-week-old culture of M. ulcerans CU001 (positive control) was extracted using a QIAamp Tissue Kit by QUIAGENBioRobot EZ1, according to the manufacturer's instructions (Qiagen, Hilden, Germany). To assess PCR inhibition, $10 \mu \mathrm{L}$ of an external control was added to $190 \mu \mathrm{L}$ of sample volume as previously described [21. Extracted DNA was used in real-time PCR (RT-PCR) to amplify the insertion sequences (IS2404 and IS2606) and the ketoreductase-B domain of the mycolactone polyketide synthase genes (KR-B) [22] using RT-PCR reagents from Roche PCR Kit (Roche Diagnostics, Meylan, France) and primers and probes as previously described [21] in a CFX 96 ${ }^{\mathrm{TM}}$ real-time PCR thermocycler and detection system (BIO-Rad, Marnes-la-Coquette, France). To estimate $M$. ulcerans inoculum in skin swabs, we performed three calibration curves for the IS2404, IS2606, and KR-B genes. The total DNA of the $M$. ulcerans CU001 strain calibrated at 1 
129 McFarland $=10^{6} \mathrm{CFU}$ was extracted with EZ (Qiagen, Hilden, Germany). Then, 10-fold serial

130 dilutions of up to $10^{-8}$ were made to generate a calibration curve for each system. Two reactional

131 mixes were incorporated into each PCR run as negative controls. Samples were considered

132 positive when the KR-B gene was detected with $\mathrm{Ct}<40$ cycles, and at least one of the two PCRs

133 of the insertion sequence gave $\mathrm{Ct}<40$ cycles and $\mathrm{KR}-\mathrm{B}$ was detected with $\mathrm{Ct}<40$ cycles.

\section{Skin microbiota repertoire.}

135 Bacterial culture. All RT-PCR-positive samples collected in Sindou were selected for culture. Six

136 negative samples collected in the same region were used as negative controls. For each sample, a cascade

137 dilution (up to $10^{-10}$ ) was performed in sterile PBS (Thermo Fisher Diagnostics, Dardilly, France), and

$138100 \mu \mathrm{L}$ of each dilution was inoculated in duplicate on blood agar plates (bioMérieux, Marcy l'Etoile,

139 France). One blood agar plate was incubated aerobically, and the second was anaerobically incubated at

$14037^{\circ} \mathrm{C}$ for 72 hours. After incubation, each colony presenting a unique morphology was sub-cultured onto

141 a blood agar plate and incubated at $37^{\circ} \mathrm{C}$ for 48 hours under the appropriate atmosphere to isolate each

142 colony type.

143 Fungal culture. $M$. ulcerans PCR-positive samples were cultured in three different culture media,

144 namely, homemade RMI medium, Sabouraud agar (Oxoid, Dardilly, France) and Chromagar (Becton

145 Dickinson, Le Pont de Claix, France) incubated at $30^{\circ} \mathrm{C}$ for seven days. Each colony was sub-cultured

146 and incubated under the same conditions. The six PCR-negative samples (negative controls) were treated

147 using the same protocol.

148 Matrix assisted laser desorption ionization time-of-flight identification of colonies. The

149 identification of the bacterial and fungal colonies was carried out using matrix-assisted laser

150 desorption ionization time-of-flight mass spectrometry MALDI-TOF-MS as previously described

151 [23,24]. For bacteria, each colony was deposited in duplicate onto a MALDI-TOF MSP 96 target

152 plate (Bruker Daltonics, Leipzig, Germany), and $2 \mu \mathrm{L}$ of matrix solution (saturated solution of 
153 alpha-cyano-4- hydroxycinnamic acid in 50\% acetonitrile and $2.5 \%$ trifluoroacetic acid) was

154 added to each spot and allowed to dry for 5 mins and then analysed by Microflex spectrometer

155 (Bruker Daltonics) using the software MALDI BioTyper 3.0 (Bruker Daltonics). For the

156 identification of fungi, each colony was incubated in $1 \mathrm{~mL}$ of $70 \%$ ethanol for $10 \mathrm{~min}$ and then

157 centrifuged at $1,300 \mathrm{~g}$ for $5 \mathrm{~min}$. The pellet was treated with $20 \mu \mathrm{L}$ of acetonitrile and formic acid

158 (v.v) at $100 \%$ and $70 \%$, respectively. This mixture was then centrifuged at $1300 \mathrm{~g}$ for $5 \mathrm{~min}$, and

$159 \quad 1.5 \mu \mathrm{L}$ of the supernatant was deposited on a MALDI-TOF-MS target and allowed to dry before

$1601.5 \mu \mathrm{L}$ of matrix was deposited on each spot, allowed to dry for $5 \mathrm{~min}$ and then analysed by par

161 Microflex spectrometer (Bruker Daltonics) using the software MALDI BioTyper 3.0 (Bruker

162 Daltonics).

163 Molecular identification and sequencing. All colonies that remained unidentified by MALDI-TOF-MS

164 were subjected to molecular identification by sequencing the bacterial 16S rRNA [25] and the fungal

165 ITS1, ITS2, $\beta$ tubulin and TEF regions. The primers used in this study are reported in Table S1. DNA

166 extraction was performed using BioRobot EZ1 (Qiagen, Les Ullis, France) using the commercial EZ1

167 DNA Tissue Kit according to the manufacturer's instructions (Qiagen). PCR was performed using

168 Hotstar Taq polymerase according to the manufacturer's instructions (Qiagen) using a thermocycler

169 (Applied Biosystem, Paris, France). PCR products were separated by electrophoresis on a $1.5 \%$ agarose

170 gel and stained with SYBR® safe (Thermo Fisher Scientific) before being visualized under an ultraviolet

171 transilluminator. PCR products were then purified using a Millipore NucleoFast 96 PCR kit following

172 the manufacturer's recommendations (Macherey-Nagel, Düren, Germany) and sequenced using the

173 BigDye Terminator Cycle Sequencing Kit (Applied Biosystems) with an automatic sequencer ABI

174 (Applied Biosystems). Sequences were assembled using the software ChromasPro 1.7 (Technelysium

175 Pty Ltd., Tewantin, Australia) and blasted in the NCBI databank (http://blast.ncbi.nlm.nih.gov/Blast.cgi) 
176 to identify bacterial species and against the Mycobank database http://www.mycobank.org/ to identify

177 fungal species.

178 Fungi-M. ulcerans biological interactions. The three fungal species found to significantly correlate

179 with the presence / absence of $M$. ulcerans skin carriage (A. flavus, A. niger and P. rubens) were cultured

180 in the presence / absence of mycolactones $\mathrm{AB} / \mathrm{C}$ extracted from a culture of $M$. ulcerans CU001 to

181 observe the effect of mycolactones on spore germination and the fungal network. Briefly, agar plates

182 were cut into T-shape, the fungal spores were placed on the middle strand, and two virgin absorbent

183 paper discs were placed on each side of the plates. 20uL of PBS were put on the first disc (negative

184 control) and 20uL of mycolactones $\mathrm{AB} / \mathrm{C}$ on the second disc. The chemoattractant effect of

185 mycolactone $\mathrm{AB} / \mathrm{C}$ was measured as previously described [25]. On the other hand, fungal spores were

186 put in Sabouraud liquid medium containing 20uL of mycolactones AB / C or 20uL of PBS as a negative

187 control. The effect of Mycolactone $\mathrm{AB} / \mathrm{C}$ on spore germination was then monitored as previously

188 described [26].

189 Statistical analyses.

190 The first exploratory step of unsupervised analysis of the cutaneous bacterial and fungal

191 microbiota used a main component analysis by integrating the presence or absence of each of the

192 fungi detected in at least one individual and adding the variable "detection of $M$. ulcerans by

193 PCR". We complemented this analysis with a supervised comparison between individuals

194 positive and negative for $M$. ulcerans by PCR. To do this, we compared the detection frequency

195 in the two groups. Statistical significance was calculated by the two-sided exact Fisher's test.

196 Owing to the possible risk of missing important findings, adjustments for multiple comparisons

197 were not performed, as suggested for exploratory work [27]. A double-clustering heatmap was

198 used to visualize the potential clustering of cultured fungal and bacterial repertoires with $M$.

199 ulcerans-positive or negative skin samples. Finally, we carried out a discriminant factor analysis 
to test whether the fungal skin microbiota discriminated against individuals who were positive or negative for $M$. ulcerans skin carriage. The principal component and discriminant factor analyses were performed using XLSTAT v2019.1 statistical and data analysis solution (Long Island, NY, USA (https://www.xlstat.com)). Multivariate analysis was performed via the Genmod procedure with the SAS 9.4 statistical software using a negative binomial distribution, adjusted for the effect of time (quantitative variable) and by using generalized estimating equations to account for the non-independence of repeated measures to analyse the results of germination assays. The

Wilcoxon signed rank test and Chi-squared test were used to compare the attraction effect of

210 https://biostatgv.sentiweb.fr.

\section{Results}

M. ulcerans DNA detection. Negative controls introduced in every batch of real-time PCR remained negative, and positive controls were positive for all the targeted sequences IS2404, IS2606 and KR-B, allowing the establishment of calibration curves. Of the 52 swabs collected in the region of Sindou, eight were positive for IS2404, six were positive for IS2606, two were positive for both IS2404 and IS2606, one was positive for both IS2404 and KR-B, two were positive for both IS2606 and KR-B, one was positive for all three targeted sequences and 32 samples were negative (Fig. 1). Therefore, 6/52 (11.5\%) swabs were positive for at least two M. ulcerans DNA sequences. As expected, only three samples were positive for IS2404 only in Tenkodogo, so none of the 52 skin samples collected in this negative control region were positive for $M$. ulcerans, confirming the probable absence of $M$. ulcerans in this region. The 
222 significant $(\mathrm{P}=0.04, \mathrm{~N}-1$ Chi-squared test $)$. Based on the calibration curves established during this

223 study, the Ct values observed here were extrapolated to 9-50 colony forming units (CFU) of M. ulcerans.

224 Skin microbiota identification. A total of 84 different species of microorganisms (62 bacterial and 22

225 fungal species) belonging to 45 different genera were recovered from the twelve samples. Thirty-one

226 different species of microorganisms (19 bacterial and 12 fungal species) grew exclusively in $M$.

227 ulcerans-PCR positive samples. Thirty-two different species (22 bacterial and 10 fungal species) grew

228 only from $M$. ulcerans-PCR negative samples. Twenty-one bacterial species grew on both $M$. ulcerans-

229 PCR positive samples and M. ulcerans-PCR negative samples. No fungal species were found in both

230 types of samples simultaneously (Fig. 2). Globally, the microbiota of M. ulcerans-PCR positive samples

231 was statistically enriched for Actinobacteria (Fisher's test p-value =0.03). The genus Aspergillus and the

232 species Aspergillus flavus were significantly associated with M. ulcerans-PCR positive samples (two-

233 sided Fisher's exact test p-value $=0.0021$ and 0.015 , respectively), and the genus Penicillium was

234 significantly associated with $M$. ulcerans-PCR negative samples (p-value $=0.0021$, Fisher's exact test).

235 All Zygomycetes, Acidovorax, Brevundimonas, Cutibacterium, and Homoserinibacter species were

236 recovered from PCR M. ulcerans-positive samples, whereas all Penicillium, Cellulosimicrobium,

237 Franconibacter, Ochrobactrum, Porphyromonas, Roseomonas, Achromobacter and Lelliottia species

238 were recovered from $M$. ulcerans-PCR-negative samples, but these associations were not statistically

239 significant (Fig. 2). The dendrogram obtained by the agglomerative hierarchical classification of the

240 species recovered from samples harboured two distinct clusters perfectly separating the M. ulcerans-

241 PCR positive samples from the M. ulcerans-PCR negative samples (Fig. 3). These results highly suggest

242 that the skin microbiota was significantly correlated with M. ulcerans carriage.

243 Principal component analysis. After submission of the results of fungal and bacterial

244 culturomics and PCR M. ulcerans to PCA analysis, two varifactors (F1 and F7; representing both

245 a total of $28.3 \%$ of the variance of the data) were selected because these two varifactors 
represented the highest variance of the variable "PCR M. ulcerans". F1 and F7 contributed 20.4\% and $7.9 \%$ of the overall variability, respectively. Aspergillus flavus, Propionimicrobium lymphophilum and Propionimicrobium freudenreichii were strongly associated with a positive

PCR for M. ulcerans, whereas Penicillium rubens, Penicillium chrysogenum and Lelliota nimipressuralis were associated with a negative PCR. Surprisingly, detection of Aspergillus fungi

positively correlated with PCR detection of M. ulcerans. Indeed, A. flavus was the fungus

exhibiting the strongest positive association with M. ulcerans. On the other hand, and ulcerans (Fig. 4).

Factor discriminant analysis. Interestingly, factor discriminant analysis identified five fungal samples, including A. flavus, Brevundimonas diminuta, Propionimicrobium lymphophilum, Pantoea dispersa and $P$. rubens (p-values: 0.001, 0.01, 0,049, 0.049, 0.049, respectively -Table operator curve analysis: area under curve $=1$ - no error in the confusion matrix - perfect clustering).

Fungi-M. ulcerans biological interactions. Mycolactones significantly increased spore germination of 
(Fig. 5). Moreover, we observed that mycolactones significantly attracted A. flavus and A. niger during the T-test assay ( $P$-value: 0.013 and 0.003 , respectively) (Fig. 6).

\section{DISCUSSION}

273 Using a standard RT-PCR assay to detect the asymptomatic skin carriage of $M$. ulcerans in skin samples

274 collected from individuals living in Burkina Faso, we detected a prevalence of $11.5 \%$ of $M$. ulcerans

275 carriage in individuals residing in the Sindou region, Burkina Faso, where Buruli ulcer cases have been

276 previously reported [20]. Our data agree with a previous report of cutaneous carriage of M. ulcerans in

277 Ghana among people practising agriculture without any protective clothing and in infants crawling on

278 the soil [28]. Thus, the data reported here confirm that in Buruli ulcer-endemic areas, some people are

279 asymptomatic skin carriers of $M$. ulcerans. The $M$. ulcerans inoculum we detected on the skin has been

280 reported to be sufficient to initiate Buruli ulcer lesions in a murine model [29]. These observations

281 suggest that asymptomatic skin carriage could be a previously undescribed condition in the natural

282 history of Buruli ulcer.

Exploration of the skin microbiota at the interface of healthy and diseased skin is in its infancy, especially regarding Buruli ulcer [30]. Comparison of skin microbiota between M. ulcerans-PCR positive and negative samples revealed a specific cutaneous microbiota associated with asymptomatic $M$. ulcerans skin carriage, even predicting M. ulcerans skin carriage. Other skin pathogens and diseases

287 (leprosy and psoriasis) have been previously related to a specific skin microbiota [31-32]. The novelty in our study was to find significant, antiparallel associations between M. ulcerans and fungi: M. ulcerans was detected on the skin along with Aspergillus, in which spore germination and the fungal network were stimulated by mycolactones, whereas the detection of $M$. ulcerans has never been associated with 
293 transkingdom mycobacteria-fungi interactions, supporting preliminary observations made with the 294 Zygomycete Mucor circinelloides [26]. Of note, the transkingdom communication concerned $P$. rubens, 295 a penicillin-producing strain, alias P. chrysogenum, made famous by Sir Alexandre Fleming [33].

296 All these observations suggest for the first time that transkingdom communications between fungi 297 and mycobacteria are of medical interest, partially driving the natural history of Buruli ulcer. These

298 observations will stimulate additional studies to disclose whether this holds true for other mycobacterial 299 infections of medical interest.

300 


\section{REFERENCES}

1. MacCallum P, Tolhurst JC, Buckle G, Sissons HA. A new mycobac- terial infection in man. J Pathol Bacteriol. 1948; 60:93-102. https://doi.org/10 .1002/path.1700600111.

2. Johnson PD, Stinear T, Small PL, Pluschke G, Merritt RW, Portaels F, et al. Buruli ulcer (M. ulcerans infection): new insights, new hope for disease control. PLoS Medicine 2005; 2:e108.

3. Brou T, Broutin H, Elguero E, Asse H, Guegan JF. Landscape diversity related to Buruli ulcer disease in Cote d'Ivoire. PLoS Negl Trop Dis. 2008; 2:e271.

4. George KM, Chatterjee D, Gunawardana G, Welty D, Hayman J, Lee R, et al. Mycolactone: a polyketide toxin from Mycobacterium ulcerans required for virulence. Science $1999 ; 283: 854-7$. plasmid-encoded polyketide synthases produce the macrolide toxin of Mycobacterium ulcerans. Proc Natl Acad Sci USA 2004; 101:1345-49. evolution and niche adaptation inferred from the genome of Mycobacterium ulcerans, the causative agent of Buruli ulcer. Genome Res. 2007; 17:192-200. mycolactone-producing mycobacteria should be considered a single species. PLoS Negle Trop Dis. 2010; 7:e663. 
8. Yoshida M, Nakanaga K, Ogura Y, Toyoda A, Ooka T, Kazumi Y. Complete Genome Sequence of Mycobacterium ulcerans subsp. shinshuense. Genome Announc. 2016; 4:1050-16.

9. Doig KD, Holt KE, Fyfe JA, Lavender CJ, Eddyani M, Portaels F, et al. On the origin of Mycobacterium ulcerans, the causative agent of Buruli ulcer. BMC Genomics. 2012; $13: 258$.

10. Ucko M, Colorni A, Kvitt H, Diamant A, Zlotkin A, Knibb WR. Strain variation in Mycobacterium marinum fish isolates. Appl Environ Microbiol. 2002; 68:5281-7.

11. Stragier P, Hermans K, Stinear T, Portaels F. First report of a mycolactone-producing Mycobacterium infection in fish agriculture in Belgium. FEMS Microbiol Lett. 2008; 286:93-5.

12. Gehringer M, Altmann KH. The chemistry and biology of mycolactones. Beilstein J Org Chem. 2017; 13:1596-660.

13. Mve-Obiang A, Lee RE, Portaels F, Small PL. Heterogeneity of mycolactones produced by clinical isolates of Mycobacterium ulcerans: implications for virulence. Immun Infect. $2003 ; 71: 774-83$.

14. George KM, Pascopella L, Welty DM, Small PL. A Mycobacterium ulcerans toxin, mycolactone, causes apoptosis in guinea pig ulcers and tissue culture cells. Infect Immun. 2000; 68:877-83.

15. Morris A L, Guégan JF, Andreou D, Marsollier L, Carolan K, Le Croller M, et al. Deforestation-driven food-web collapse linked to emerging tropical infectious disease, Mycobacterium ulcerans. Sci Adv. 2016; 2:1600387. 
16. Zingue D, Bouam A, Tian RBD, Drancourt M. Buruli ulcer, a prototype for ecosystemrelated infection, caused by Mycobacterium ulcerans. Clin Microbiol Rev. 2018; 31:4517.

17. Van Leuvenhaege C, Vandelannoote K, Affolabi D, Portaels F, Sopoh G, de Jong BC, et al. Bacterial diversity in Buruli ulcer skin lesions: Challenges in the clinical microbiome analysis of a skin disease. PloS One 2017; 12:0181994. nasal cavity colonization: role of staphylococcal agr alleles. Appl Environ Microbiol. 2003; 69:18-23. diversity of Staphylococcus aureus in Buruli ulcer. PLoS Negl Trop Dis. 2015; 9:3421. Faso: apropos of 6 cases. Tunis Med. 1998; 76:46-50. bacteriophages as molecular diagnosis controls in clinical virology: a comprehensive and application of two multiplex real-time PCR assays for the detection of Mycobacterium ulcerans in clinical and environmental samples. Appl Environ Microbiol. 2007; 73:4733-40. spectrometry applications in clinical microbiology. Future Microbiol. 2010; 5:1733-54. 

TOF MS platforms for fungal identification. Mycoses 2016; 59:678-90. $28: 380-2$.

26. Hammoudi N, Cassagne C, Armstrong N, Ranque S, Henrissat B, Drancourt M et al. Mycobacterium ulcerans mycolactones-fungi crosstalking. Sci Rep. 2019; 9:3028. $1990 ; 43-46$.

28. Prah I. Examination of human skin surfaces for the detection of Mycobacterium ulcerans. M.Sc. Thesis. University of Ghana. 2015. Available from: http://ugspace.ug.edu.gh/handle/123456789/8369

29. Wallace JR, Mangas KM, Porter JL, Marcsisin R, Pidot SJ, Howden B et al. Mycobacterium ulcerans low infectious dose and mechanical transmission support insect bites and puncturing injuries in the spread of Buruli ulcer. PLoS Negl Trop Dis. 2017;

30. Cho I, Blaser M J. The human microbiome at the interface of health and disease. Nat Rev Genet. 2012; 13:260-70. the skin microbiome dynamics of leprosy patients during multi-drug therapy and in in skin biopsies from normal and psoriatic skin. Arch Dermatol Res. 2012; 304:15-22. 
bioRxiv preprint doi: https://doi.org/10.1101/869636; this version posted December 10, 2019. The copyright holder for this preprint (which was not certified by peer review) is the author/funder, who has granted bioRxiv a license to display the preprint in perpetuity. It is made available under aCC-BY-NC-ND 4.0 International license. 
391 The authors acknowledge the technical help of Nicholas Armstrong.

392 This work was supported by the French Government under the «Investissements d'avenir »

393 (Investments for the Future) programme managed by the Agence Nationale de la Recherche

394 (ANR, fr: National Agency for Research), (reference: Méditerranée Infection 10-IAHU-03). This

395 work was supported by Région Sud (Provence Alpes Côte d'Azur) and European funding

396 FEDER PRIMMI. 


\section{FIGURE LEGENDS}

399 Fig. 1: Real-time PCR detection of M. ulcerans from 52 swabs collected in the Sindou region,

400 IS2404 and IS2606 are specific insertion sequences for M. ulcerans KR-B ketoreductase-B gene.

401 Fig. 2: The different microorganisms grown from 12 skin samples comprising 6 M. ulcerans

402 positive samples and 6 negative samples are presented with their relative frequency differences

403 and associated P-values. A total of 84 species distributed among 62 bacteria and 22 fungi were

404 cultured.

405 Fig. 3: The agglomerative hierarchical classification dendrogram of individuals according to their

406 cutaneous microbiota associated with the detection (P1 to P6) or absence (N1 to N6) of $M$.

407 ulcerans. M. ulcerans PCR-negative samples and M. ulcerans PCR-positive samples were clearly

408 separated into two distinct clusters according to the composition of the skin microbiota.

409 Fig. 4: PCA of 84 bacterial and fungal species isolated from individuals in Burkina Faso revealed

410 a strong association of $A$. flavus, P. lymphophilum and P. freudenreichii with $M$. ulcerans skin

411 carriage.

412 Fig. 5: Mycolactone-induced spore germination of A. flavus, A niger and P. rubens in the

413 presence of PBS as a negative control.

414 Fig. 6: Fungi attraction test: mycolactones (right side disk) attract A. flavus and A. niger in the 415 presence of PBS as a negative control (left side disk). 


\section{Supplementary data}

417 Supplementary Table 1: Primers used in this study for the PCR-based identification of fungi and 418 bacteria.

\begin{tabular}{|c|c|c|}
\hline & Name & Sequences 5'-3' \\
\hline \multirow{10}{*}{$\begin{array}{c}\text { Fungal } \\
\text { sequences }\end{array}$} & ITS3 & GCATCGATGAAGAACGCAGC \\
\hline & ITS4 & TCCTCCGCTTATTGATATGC \\
\hline & $\mathrm{Bt} 2 \mathrm{a}$ & GGTAACCAAATCGGTGCTGCTTTC \\
\hline & $\mathrm{Bt} 2 \mathrm{~b}$ & ACCCTCAGTGTAGTGACCCTTGGC \\
\hline & $\mathrm{Al} 33 \mathrm{~F}$ & GAYTTCATCAAGAACATGAT \\
\hline & A133R & GACGTTGAADCCRACRTTGTC \\
\hline & $\mathrm{A} 134 \mathrm{~F}$ & TTCATCAAGAACATGAT \\
\hline & $\mathrm{Al} 34 \mathrm{R}$ & GCTATCATCACAATGGACGTTCTTGGAG \\
\hline & ITS1 & TCCGTAGGTGAACCTGCGG \\
\hline & ITS2 & GCTGCGTTCTTCATCGATGC \\
\hline \multirow{8}{*}{$\begin{array}{l}\text { Bacterial } \\
\text { sequences }\end{array}$} & FD1 & AGAGTTTGATCCTGGCTCAG \\
\hline & $\mathrm{rP} 2$ & ACGGCTACCTTGTTACGACTT \\
\hline & $536 \mathrm{~F}$ & CAGCAGCCGCGGTAATAC \\
\hline & $536 \mathrm{R}$ & GTATTACCGCGGCTGCTG \\
\hline & $800 \mathrm{~F}$ & ATTAGATACCCTGGTAG \\
\hline & $800 \mathrm{R}$ & CTACCAGGGTATCTAAT \\
\hline & $1050 \mathrm{~F}$ & TGTCGTCAGCTCGTG \\
\hline & 1050R & CACGAGCTGACGACA \\
\hline
\end{tabular}


421 Supplementary Table 2: Factor discriminant analysis (FDA) revealed five fungal and bacterial

422 species that significantly discriminated M. ulcerans-PCR positive and negative samples.

423

\begin{tabular}{|l|c|c|c|c|c|}
\hline Variable & Lambda & F & DDL1 & DDL2 & p-value \\
\hline Propionimicrobium lymphophilum-0 & 0,667 & 5,000 & 1 & 10 & 0,049 \\
\hline Brevundimonas diminuta-0 & 0,500 & 10,000 & 1 & 10 & 0,010 \\
\hline Pantoea dispersa-0 & 0,667 & 5,000 & 1 & 10 & 0,049 \\
\hline Penicillium rubens-0 & 0,667 & 5,000 & 1 & 10 & 0,049 \\
\hline Aspergillus flavus-0 & 0,286 & 25,000 & 1 & 10 & 0,001 \\
\hline
\end{tabular}

424

425 
M. ulcrans positves sampls

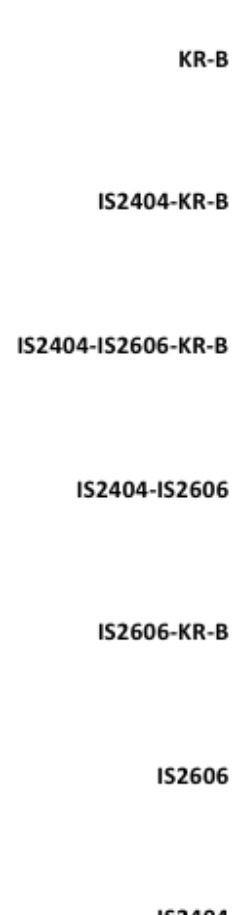

Fig. 1. 
Propionimicrobium lymphophilum

Bacillus cereus

Microbacterium paraoxydans

Lactobacillus plantarum

Bacillus dielmoensis

Bacillus megaterium

Microbacterium oleivorans

Propionibacterium freudenreichii

Micrococcus luteus

Clostridium subterminale

Stenotrophomonas maltophila

Pseudomonas aeruginosa

Pantoea dispersa

Bacillus marasmi

Paenibacillus alvei

Staphylococcus capitis

Lactobacillus reuter

Bacillus pumilus

Pichia carribica

Cutibacterium acnes

Microbacterium hydrocarbonoxydans

Microbacterium testaceum

Homoserinibacter gongjuensis

Purpureocillium lilacinum

Aspergillus nidulans

Aspergillus tubingensis

Curtobacterium citreum

Microbacterium arborescens

Ruminococcus merdae

Lodderomyces elongisporus

Arxula adeninivorans

Ogataea thermomethanolica

Exophiala spinifera

Syncephalastrum racemosum

Rhizopus oryzae

Acinetobacter Iwoffii

Microbacterium lacticum

Cellulomonas parahominis

Fig. 2. Microbacterium neinengenese

Collinsella massiliensis

Klebsiella pneumoniae

Enterobacter cloacae

Propionibacterium acnes

Cryptococcus laurentii

Gymnascella aurantiaca

Pochonia chlamydosporia

Exophiala xenobiotica

Sordariomycete $s p$

Penicillium commune

Dothideomycete

Microbacterium paludicola

Cellulomonas iranensis

Cellulosimicrobium cellulans

Franconibacter pulveris

Pantoea terrea

Pantoea anthophila

Ochrobactrum haemophilum

Roseomonas gilardii

Pseudomonas stutzeri

Pantoea calida

Bacillus benzoevorans

Bacillus niacini

Bacillus subtilis

Bacillus drentensis

Bacillus paralicheniformis

Porphyromonas uenonis

Penicillium crustosum

Micrococcus yunnanensis

Achromobacter spanius

Paenibacillus yonginensis

Bacillus marisflavi

Staphylococcus hominis

Penicillium rubens

Penicillium chrysogenum

Pantoea stewartii

Lelliottia nimipressuralis 


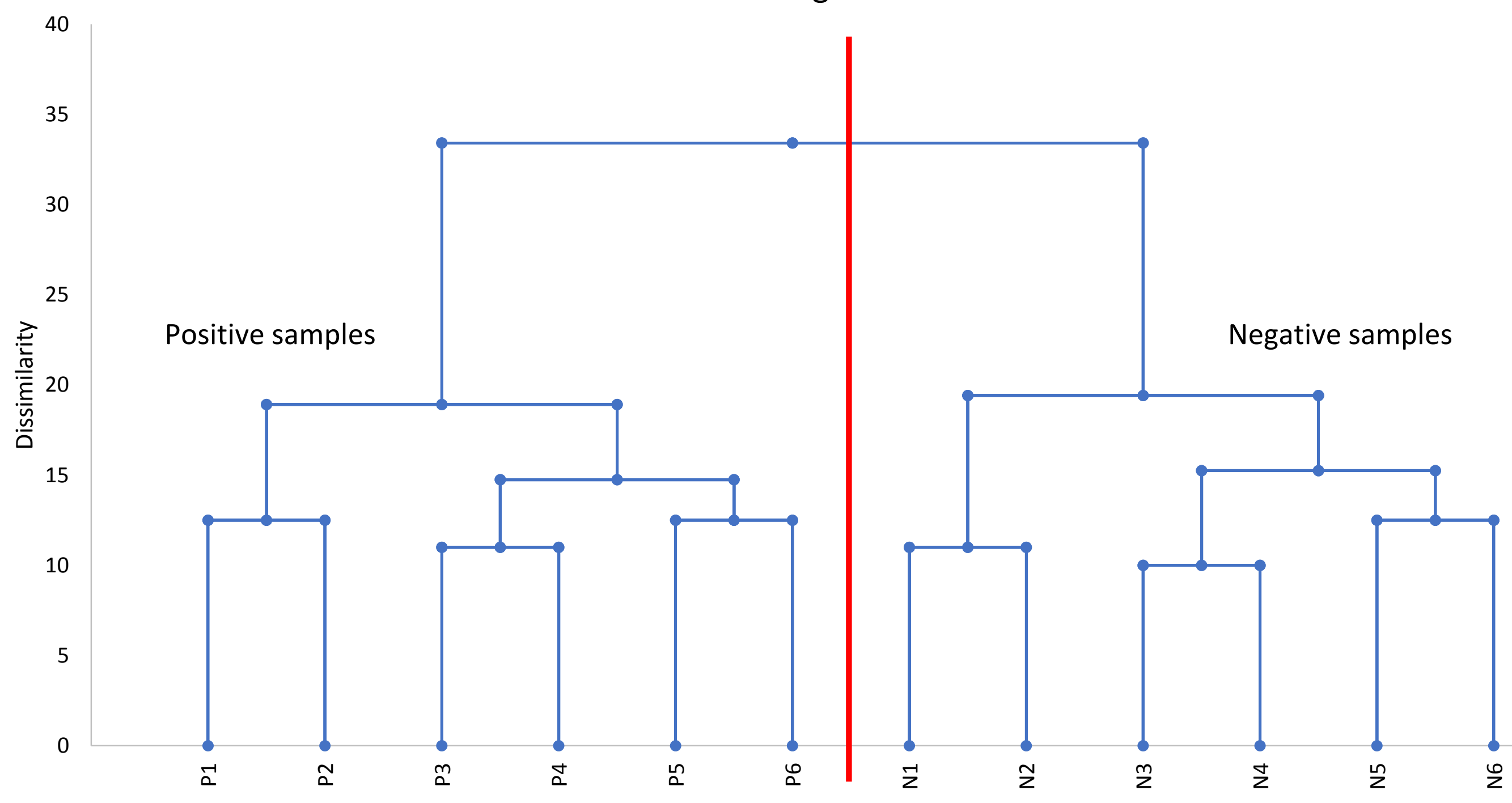

Fig. 3. 
0.75

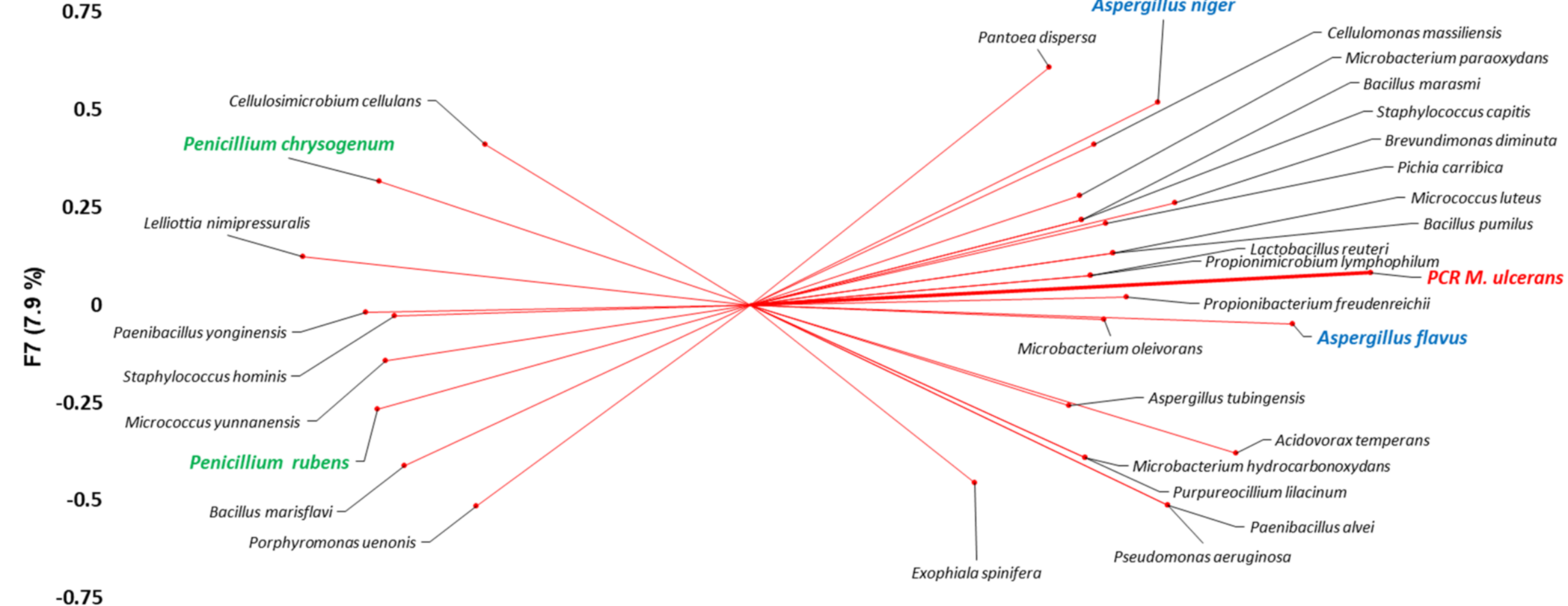

$-1$

$-1$

$-0.75$

0

0.25

0.5

0.75

1

Fig. 4.

F1 (20.4\%) 

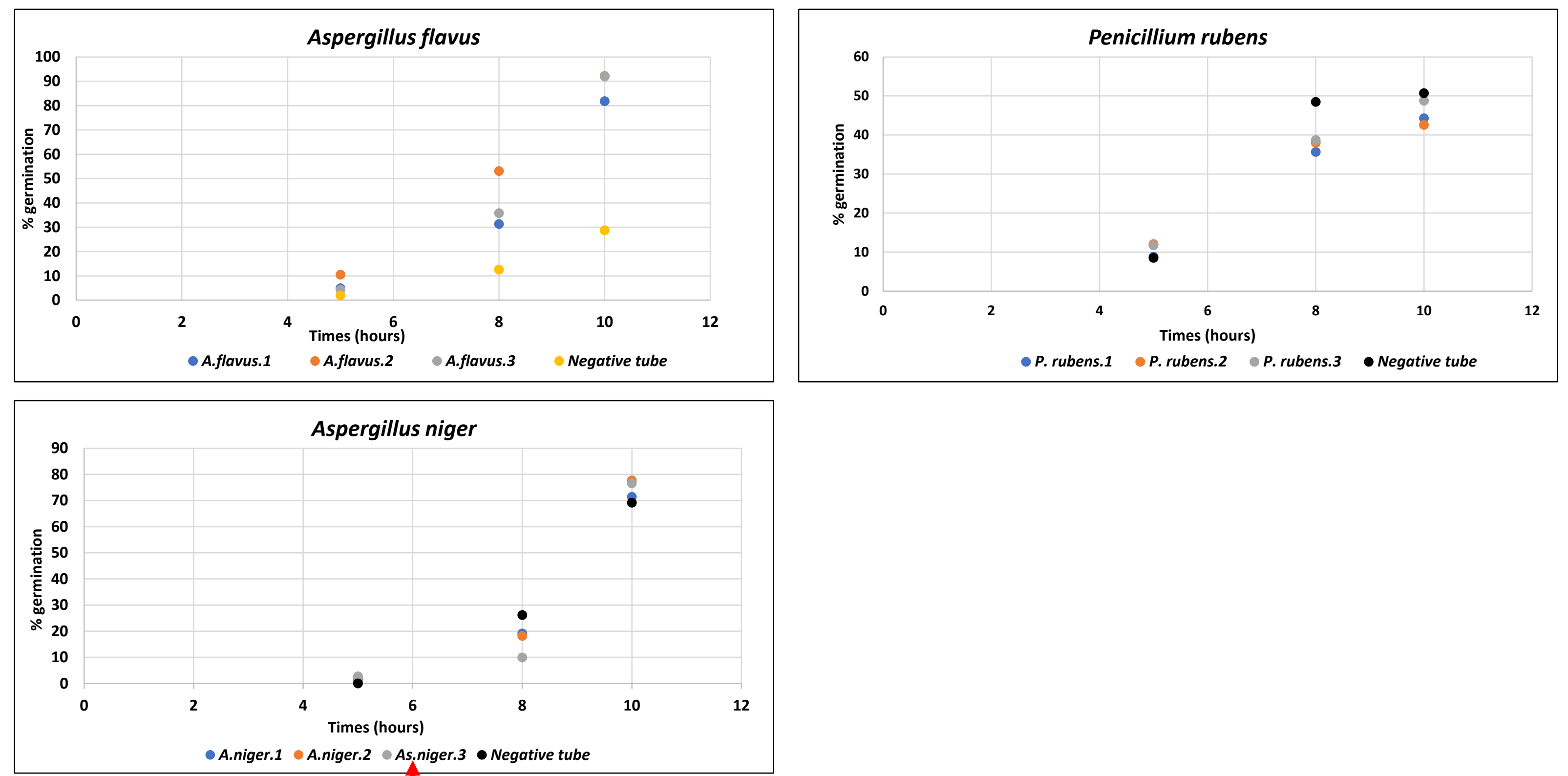

Fig. 5. 

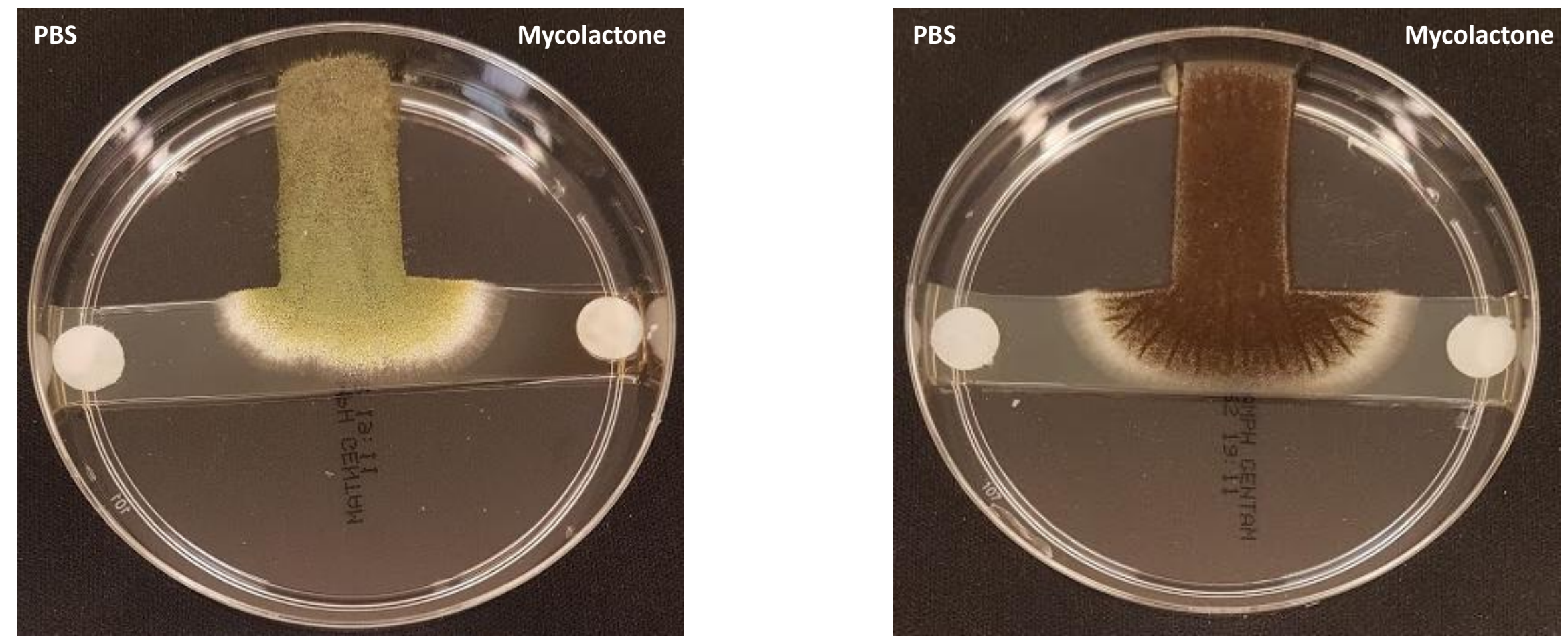

Fig. 6. 